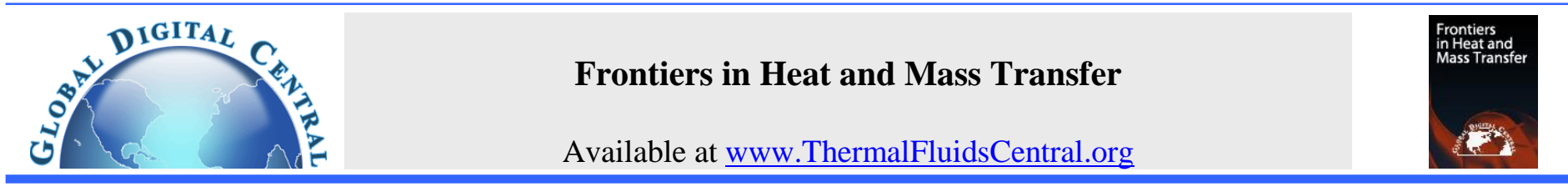

\title{
INVESTIGATION ON CNTS-WATER AND HUMAN BLOOD BASED CASSON NANOFLUID FLOW OVER A STRETCHING SHEET UNDER IMPACT OF MAGNETIC FIELD
}

\author{
${ }^{a}$ Department of Mathematics, Faculty of Science, Ajloun National University, \\ P.O. Box 43, Ajloun 26810, Jordan \\ ${ }^{b}$ Faculty of Arts and Sciences, Aqaba University of Technology, Aqaba-Jordan \\ ${ }^{c}$ Department of Mathematics, Al-Hussein Bin Talal University,Ma'an-Jordan
}

Hamzeh T. Alkasasbeh ${ }^{a^{*}}$, Mohammed Z. Swalmeh ${ }^{\mathrm{b}}$, Hebah G. Bani Saeed $^{\mathrm{c}}$, Feras M. Al Faqih ${ }^{\mathrm{c}}$, Adeeb G. Talafha ${ }^{\mathrm{c}}$

\begin{abstract}
This study aims at considering the properties of heat transfer and magneto-hydrodynamics (MHD) Casson nanofluid at the existence of free convection boundary layer flow with Carbon Nanotubes (CNTs) suspended in human blood/water as based fluid on a stretching sheet. Two types of CNTs nanoparticles, single walled carbon nanotubes (SWCNTs) and multi walled carbon nanotubes (MWCNTs), are taken into account. The governing partial differential equations are transformed to partial differential equations using similar transformation, then solved numerically by an implicit finite difference scheme known as Keller-box method (KBM). The results for physical quantities, the local skin friction, and local Nusselt number, as well as temperature and velocity, are discussed under the magnetic nanofluid Casson parameters. This work is compared with recently published results on the Newtonian fluid as a special case and shows very good agreement.
\end{abstract}

Keywords: Casson Nanofluid fluid; MHD, Stretching Sheet; SWCNTs and MWCNTs.

\section{INTRODUCTION}

Casson fluid is a subcategory of Non-Newtonian fluids as toothpaste, food materials, and plastic has several utilizes in medical chemical industries and cosmetics. It is hard to handle this type of fluid, because it behaves as an elastic solid, such that no flow happens with short shear stress. Hence, different fluid models are showed to characterize the effectiveness of the materials. In the current consideration, we investigate the Casson model. This model was first conducted by Casson (1959). After that, many types of researches had been conducted in the same field. Mustafa et al. (2012); Mustafa et al. (2011) investigated the parallel free stream in the presence of the Casson fluid on a semi-infinite flat plate and in the region of stagnation point towards a stretching sheet. Nadeem and UI (2013) studied the boundary layer three- dimensional flow of Casson fluid on a stretching sheet. Another Various researches on Casson fluids can also be found in the literature of Hayat et al. (2012), Nadeem et al. (2013), Bhattacharyya (2013); Qing et al. (2016), Tahir et al. (2017), Alsagri et al. (2019), Hussanan et al. (2018), and Alwawi et al. (2019).

Recently, several researches have been conducted to develop the heat transfer and reach various levels of thermal competences. This technique can be used through increasing the thermal conductivity.Choi andEastman (1995) were the first who examined the enhancement of heat transfer in convectional fluids with nanoparticles, typically with a size less than $100 \mathrm{~nm}$. After that, many researchers considered it because of its thermal characteristics and its future applications. Moreover, mathematical models have been conducted to investigate in the properties of nanofluids Tiwari and Das (2007). Tiwari-Das model has succeeded in describing the behaviors of nanofluid in boundary layer flow. Besides, many researchers published various articles around the boundary layer flow in a nanofluid, such as Chamkha and Aly (2010); Chamkha et al. (2011), Bhattacharyya (2013); Chamkha (2011), Mukhopadhyay et al. (2013) and Swalmeh et al. (2019 a,b).

It is said that human blood is an example of Casson fluid, and many studies used physical properties of the human blood as based fluid under boundary layer flow, see Khalid et al. (2018), Rashidi et al. (2016), and Alsagri et al. (2019). CNTs (carbon nanotubes) have a long cylindric shape, such as structures of carbon atoms with sizes typically of 0.7-50 nm. In 1991, the concept of CNTs was first investigated by Iijima (1991). Carbon nanotubes are split into two types, namely single wall carbon nanotubes (SWCNTs), and multi wall carbon nanotubes (MWCNTs), relying upon concentric layers of rolled graphene lamina. Moreover, carbon nanotubes are expected to be a creative matter on our time because of their industrial and mechanical applications. There are many studies that take the CNTs nanoparticles into consideration. Haq et al.(2015) considered the effects of the thermo-physical characteristics of CNTs nanoparticles based nanofluids on a stretching surface. The heat transfer flow of nanofluids including CNTs on a flat plate subjected to the Navier slip boundary condition, and the impacts of (MHD) flow on of non-Newtonian as CNTs nanofluid were investigated by Khan et al. (2014), and Aman et al. (2017). Several other significant kinds of researches on CNTs based nanofluid have been checked by Khalid et al. (2018); Sallam and Hwang (1984), Nasir et al. (2018), Ding et al. (2006), and Alsagri et al. (2019)

Recently, MHD free convection have had great solicitation and importance because of the high wide modern industrial implementations, and thus many articles have been published in this

*Corresponding author: hamzahtahak@yahoo.com 
field. MHD free convection with aligned magnetic radiation and variable viscosity on a semi-infinite flat plate were investigated by Seddeek (2002), and Makinde (2012) who considered the collective impacts of Navier slip and Newtonian heat on an unsteady (MHD) magnetic boundary layer flow over a flat plate. The effects of magnetohydrodynamics (MHD) heat transfer and entropy generation flow with the influence of slip-on the flat plate were studied by Ellahi et al. (2018). The (MHD) flow with suitable nanoparticles as Ag suspended in water-based nanofluid over a flat plate in a porous medium,and unsteady magneto-hydrodynamic free convection flow on an infinite vertical flat plate under the characteristics of the uniform transverse magnetic field were considered by Upreti et al. (2018), and Palani and Srikanth (2009). Another studies concerned with the MHD flow on a solid sphere and a horizontal circular cylinder, are Qadan et al. (2019), Alkasasbeh et al. (2014), Subba Rao et al. (2017) Alkasasbeh (2018), Prasad et al. (2012), Alwawi et al. (2020); and Sheikholeslami et al. (2015).

The present study has discussed the Heat and mass transfer effect of free convection on MHD Casson nanofluid over a stretching sheet subject to the constant wall temperature. At the same time, it has also used the similar transformations to transform the governing equations in the dimensional form to PDEs. The reduced PDEs are solved numerically by using Keller-box scheme. The numerical calculations for various physical parameters are carried out up to a required level of accuracy $\left(10^{-5}\right)$. The results of velocity and temperature profiles are plotted through graphs. The results of the skin friction and Nusselt number are calculated carefully and showed in a form of table.

\section{MATHEMATICAL FORMULATION}

Consider the free convection two-dimensional boundary layer flow of incompressible magnetohydrodynamic (MHD), Casson nanofluid over stretching sheet, which the flow begun at $y=0$, and being restricted in $y>0$, as presented in fig. 1 , where $u_{w}, u_{s}$ are shrinking/stretching velocity and straining velocity, respectively. Two types of Carbon Nanotubes (CNTs) nanoparticles, such as single walled carbon nanotubes (SWCNTs) and multi walled carbon nanotubes (MWCNTs), in two based fluids namely water and human blood, are investigated. The nanofluid is permeated in a Casson medium and a variable magnetic field strength $B_{0}$ and applied in the cross direction of the fluid flow. The flow is supposed to be in the $x$-direction, which is taken along the stretching sheet in the upward direction and the $y$-axis is perpendicular to it. Accordingly the same initial temperature is preserved. Instantaneously they are increased to a prescribed wall temperature $T_{w}>T_{\infty}$ the ambient temperature of the fluid that still unchanged. The impacts of nanoparticle volume fraction, Casson, magnetic, and electrical conductivity have merged in the momentum equation.

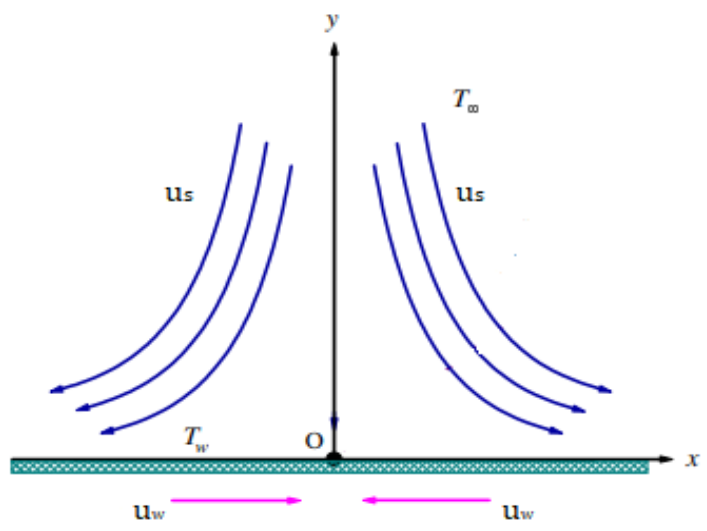

Fig. 1 Physical sketch of the problem.
The equation obtained below for determining the rheological property of Casson fluid. Casson (1959)

$$
\tau_{i j}= \begin{cases}2\left(\mu_{B}+p_{y} / \sqrt{2 \pi}\right) e_{i j} & \pi>\pi_{c}, \\ 2\left(\mu_{B}+p_{y} / \sqrt{2 \pi_{c}}\right) e_{i j} & \pi<\pi_{c},\end{cases}
$$

Here, $\pi=e_{i j} e_{i j}, e_{i j}$ is the $(i, j)$-th component of the deformation rate, $\pi_{c}, p_{y}$, and $\mu_{\beta}$ respectively are critical values of this product based on the non-Newtonian model, the yield stress of the fluid, and the plastic dynamic viscosity of the non-Newtonian fluid.

In the case of the Casson fluid, $\pi>\pi_{c}$ so:

$$
\mu=\mu_{B}+p_{y} / \sqrt{2 \pi}
$$

Substitute the value of $p_{y}=\mu_{B} \sqrt{2 \pi} / \beta$ in equation (2), then the kinematic viscosity of the Casson fluid, which $\mu_{B}$ is purely dependent on plastic dynamic viscosity, $\rho$ is density and $\beta$ is parameter of the Casson fluid as

$$
\frac{\mu}{\rho}=\frac{\mu_{B}}{\rho}+\left(1+\frac{1}{\beta}\right)
$$

Depending on the previous assumptions, the governing equations, the continuity, momentum and energy equations, for Casson nanofluid over a stretching sheet can be presented as follows:

$$
\begin{gathered}
\frac{\partial \tilde{u}}{\partial \tilde{x}}+\frac{\partial \tilde{v}}{\partial \tilde{y}}=0, \\
\tilde{u} \frac{\partial \tilde{u}}{\partial \tilde{x}}+\tilde{v} \frac{\partial \tilde{u}}{\partial \tilde{y}}=v_{n f}\left(1+\frac{1}{\beta}\right) \frac{\partial^{2} \tilde{u}}{\partial \tilde{y}^{2}}-\frac{\sigma_{n f}}{\rho_{n f}} B_{0}^{2} \tilde{u}, \\
\tilde{u} \frac{\partial T}{\partial \tilde{x}}+\tilde{v} \frac{\partial T}{\partial \tilde{y}}=\frac{K_{n f}}{\left(\rho C_{p}\right)_{n f}} \frac{\partial^{2} T}{\partial \tilde{y}^{2}}
\end{gathered}
$$

Subject to the constant wall temperature boundary condition:

$$
\begin{gathered}
\tilde{u}=u_{w}(\tilde{x})=a \tilde{x}, \tilde{v}=0, T=T_{w} \text { at } \tilde{y}=0, \\
\tilde{u} \rightarrow 0, T \rightarrow T_{\infty} \text { as } \tilde{y} \rightarrow \infty,
\end{gathered}
$$

where $\tilde{u}$ and $\tilde{v}$ are the velocity components along the $\tilde{x}$ and $\tilde{y}$ directions, respectively. The properties of nanofluid are expressed by Ahmed and Khan (2018), and Swalmeh et al. (2019 a) as follows:

$$
\begin{gathered}
\rho_{n f}=(1-\chi) \rho_{f}+\chi \rho_{s}, \mu_{n f}=\frac{\mu_{f}}{(1-\chi)^{2.5},} \\
\left(\rho c_{p}\right)_{n f}=(1-\chi)\left(\rho c_{p}\right)_{f}+\chi\left(\rho c_{p}\right)_{s}, \\
\frac{k_{n f}}{k_{f}}=\frac{\left(k_{s}+2 k_{f}\right)-2 \chi\left(k_{f}-k_{s}\right)}{\left.\left(k_{s}+2 k_{f}\right)+\chi k_{f}-k_{s}\right)}, \alpha_{n f}=\frac{k_{n f}}{\left(\rho c_{p}\right)_{n f}}, \\
\sigma_{n f}=\left[1+\frac{3\left(\left(\sigma_{s} / \sigma_{f}\right)-1\right) \chi}{\left(\left(\sigma_{s} / \sigma_{f}\right)+2\right)-\left(\left(\sigma_{s} / \sigma_{f}\right)-1\right) \chi}\right] \sigma_{f}, .
\end{gathered}
$$

Using the following similarity transformations in the governing equations (4) to (6) subject to the boundary conditions (7) Salleh et al. (2010):

$$
\begin{gathered}
\tilde{u}=\frac{\partial \psi}{\partial \tilde{y}}, \tilde{v}=-\frac{\partial \psi}{\partial \tilde{x}} \\
\psi=(a \tilde{v})^{1 / 2} \tilde{x} f(\eta), \quad \eta=(a \tilde{v})^{1 / 2} \tilde{y}, \quad \theta(\eta)=\frac{T-T_{\infty}}{T_{w}-T_{\infty}} .
\end{gathered}
$$

where $\psi$ is the stream function

By substituting the equations (6) and (7) we get the following equations

$$
\left(\frac{\rho_{f}}{\rho_{n f}} \frac{1}{(1-\chi)^{2.5}}\right)\left(1+\frac{1}{\beta}\right) f^{\prime \prime \prime}+f f^{\prime \prime}-\left(f^{\prime}\right)^{2}-\frac{\rho_{f}}{\rho_{n f}} \frac{\sigma_{n f}}{\sigma_{f}} M f^{\prime}=0
$$




$$
\frac{1}{\operatorname{Pr}}\left[\frac{k_{n f} / k_{f}}{(1-\chi)+\chi\left(\rho c_{p}\right)_{s} /\left(\rho c_{p}\right)_{f}}\right] \theta^{\prime \prime}+f \theta^{\prime}=0,
$$

and the corresponding boundary condition are

$$
\begin{gathered}
f^{\prime}=1, f=0, \theta=1 \text { as } \eta=0, \\
f^{\prime} \rightarrow 0, \theta \rightarrow 0, \text { as } \eta=\infty .
\end{gathered}
$$

Here we have $\operatorname{Pr}=\frac{v_{f}}{\alpha_{f}}$ is called the Prandtl number and $M=\left(\frac{\sigma_{f} B_{0}^{2} a^{2}}{\rho_{f} v_{f}}\right)$ is magnetic parameter.

For the engineering usefulness, the physical quantities especially, the local skin friction coefficient $\left(C_{f}\right)$ and the Nusselt number $(\mathrm{Nu})$, the model are followed respectively,

$$
C_{f}=\left(\frac{\tau_{w}}{\rho U_{w}^{2}}\right)_{\tilde{y}=0} \text {, and } N u=\left(\frac{a q_{w}}{k_{f}\left(T_{w}-T_{\infty}\right)}\right)_{\tilde{y}=0},
$$

with $\tau_{w}$ and $q_{w}$ are corresponding, shear stress, and heat flux on the plane of the wall are defined below.

$$
\tau_{w}=\mu_{n f}\left(\frac{\partial^{2} u}{\partial \bar{y}^{2}}\right)_{\tilde{y}=0} \text { and } q_{w}=-k_{n f}\left(\frac{\partial T}{\partial \tilde{y}}\right)_{\tilde{y}=0} .
$$

Now, the local skin friction coefficient and Nusselt number become as:

$$
\operatorname{Re}^{1 / 2} C_{f}=\frac{1}{(1-\chi)^{2.5}}\left(1+\frac{1}{\beta}\right) f^{\prime \prime}(0), \operatorname{Re}^{-1 / 2} N u=-\frac{k_{n f}}{k_{f}} \theta^{\prime}(0),
$$

where $\operatorname{Re}^{1 / 2}=\left(a \tilde{x}^{2} / v_{f}\right)$ is denoted the local Reynolds number.

\section{RESULTS AND DISCUSSION}

The numerical solutions obtained by using the Keller box method of the above governing equations (10) and (11) subject to the boundary condition (12) are presented in figures and tables, see Keller and Bramble (1970) and Cebeci and Bradshaw (2012). Many convection boundary layer problems use this method to study the numerical solutions and its physical quantities behaviors, this method appears to be the most malleable of the used methods and in spite of novel expansions in other numerical methods, stills a potent and very accurate process for parabolic boundary layer flows. It is also being easily adjustable to solve equations of any order and unconditionally stable on the solutions. The Keller box method procedures are: firstly, reduce PDEs to a first-order system by using a finite difference scheme. Next, Write the difference equations by using central differences, and linearize the resulting differences equations by Newton's method and write them in the matrix-vector form. Finally, solve the linear system by the block tri-diagonal elimination technique, and then writing the steps of Keller-box method in Matlab program see Swalmeh et al (2019 a).

Table 1: Different values of thermophysical properties of CNTs of two base Casson nanofluidsAlsagri et al. (2019)

\begin{tabular}{|l|l|l|l|l|}
\hline & \multicolumn{2}{|l|}{ Based fluids } & \multicolumn{2}{l|}{ CNTs nanoparticles } \\
\hline $\begin{array}{l}\text { Physical } \\
\text { properties }\end{array}$ & Water & Human blood & SWCNT & MWCNT \\
\hline $\mathrm{k}(\mathrm{W} / \mathrm{mK})$ & 0.613 & 0.492 & 6600 & 3000 \\
\hline$\rho\left(\mathrm{kg} / \mathrm{m}^{3}\right)$ & 997.1 & 1053 & 2600 & 1600 \\
\hline $\mathrm{c}_{\mathrm{p}}(\mathrm{J} / \mathrm{kgK})$ & 4179 & 3594 & 425 & 796 \\
\hline$\delta \mathrm{s}\left(\mathrm{Sm}^{-1}\right)$ & $5.5 \times 10^{-6}$ & 0.8 & $10^{6}-10^{7}$ & $1.9^{-4}$ \\
\hline $\operatorname{Pr}$ & 6.2 & 24 & & \\
\hline
\end{tabular}

Two kinds of Carbon Nanotubes (CNTs) nanoparticles, namely single and multi -walled carbon nanotubes (SWCNTs), (MWCNTs), in two based fluids namely, water and human blood. The impact of various values of nanoparticle volume fraction $0 \leq \chi \leq 0.2$, Casson $\beta \geq 1$, and magnetic parameters $M \geq 0$ on the temperature $\theta(0)$ and velocity $f^{\prime}(0)$ profiles have been outlined in figures (2-7). Meanwhile, the Nusselt number and local skin friction coefficient have been enumerated and put up in the table 3. Thermo-physical characteristics of based fluids and nanoparticles are set in table 1. Also, table 2 shows the comparison values with the previous literature stated by Hassanien et al. (1998) and Salleh et al. (2010). It is noted that the results of the present study are in a good agreement.

Table 2: Comparison of $\operatorname{Re}^{-1 / 2} N u$ with viscous Newtonian fluid ( $M=$ $0, \beta=\infty$, and $\chi=0$ ), with several values of $\operatorname{Pr}$.

\begin{tabular}{|l|l|l|l|}
\hline $\operatorname{Pr}$ & \multicolumn{3}{|l|}{$\mathrm{Re}^{-1 / 2} \mathrm{Nu}$} \\
\hline & $\begin{array}{l}\text { Hassanien } \\
\text { et al. (1998) }\end{array}$ & $\begin{array}{l}\text { Salleh et al. } \\
(2010)\end{array}$ & Present \\
\hline 0.72 & 0.46325 & 0.46317 & 0.46316 \\
\hline 1 & 0.58198 & 0.58198 & 0.58198 \\
\hline 3 & 1.16525 & 1.16522 & 1.16524 \\
\hline 5 & & 1.56806 & 1.56807 \\
\hline 7 & & 1.89548 & 1.89550 \\
\hline 10 & 2.30801 & 2.30821 & 2.30820 \\
\hline 100 & 7.74925 & 7.76249 & 7.76250 \\
\hline
\end{tabular}

Table 3: Values of the skin friction coefficient $\operatorname{Re}^{1 / 2} C_{f}$ and local Nusselt number $\operatorname{Re}^{-1 / 2} N u$ for a different values of Casson parameter $\beta, \chi$ and $M$.

\begin{tabular}{|l|l|l|l|l|l|l|}
\multicolumn{2}{c}{} & \multicolumn{3}{c}{$\begin{array}{l}\text { SWCENT } \\
\text { Human blood }\end{array}$} & \multicolumn{2}{l|}{$\begin{array}{l}\text { MWCENT } \\
\text { Human Blood }\end{array}$} \\
\hline$\beta$ & $\chi$ & $M$ & $\operatorname{Re}^{1 / 2} C_{f}$ & $\operatorname{Re}^{-1 / 2} N u$ & $\operatorname{Re}^{1 / 2} C_{f}$ & $\operatorname{Re}^{-1 / 2} N u$ \\
\hline 3 & 0.075 & 2 & -7.7591 & 5.1829 & -7.7213 & 5.1613 \\
\hline 7 & & & -16.7940 & 5.1472 & -16.682 & 5.1267 \\
\hline 10 & & & -23.5388 & 5.1381 & -23.384 & 5.1176 \\
\hline 3 & 0.1 & 5 & -11.9797 & 5.6423 & -11.818 & 5.6152 \\
\hline & 0.15 & & -14.2650 & 7.1565 & -14.027 & 7.0985 \\
\hline & 0.2 & & -17.0390 & 9.0564 & -16.734 & 8.9481 \\
\hline 3 & 0.15 & 1 & -8.2849 & 7.4889 & -8.2742 & 7.4182 \\
\hline & & 2 & -10.1148 & 7.3874 & -10.027 & 7.3211 \\
\hline & & 3 & -11.6647 & 7.3014 & -11.516 & 7.2384 \\
\hline & & 7 & -16.4589 & 7.0339 & -16.152 & 6.9798 \\
\hline
\end{tabular}

(b)

\begin{tabular}{|l|l|l|l|l|l|l|}
\cline { 4 - 7 } \multicolumn{2}{c|}{} & \multicolumn{2}{l}{$\begin{array}{l}\text { SWCENT } \\
\text { Water }\end{array}$} \\
\hline$\beta$ & $\chi$ & $\mathrm{M}$ & $\mathrm{Re}^{1 / 2} C_{f}$ & $\mathrm{Re}^{-1 / 2} N u$ & \multicolumn{2}{l|}{$\begin{array}{l}\text { WWCENT } \\
\text { Water }\end{array}$} \\
\hline 3 & 0.075 & 2 & -7.8666 & 2.4157 & -8.6175 & 2.3634 \\
\hline 7 & & & -16.997 & 2.3761 & -18.616 & 2.3247 \\
\hline 10 & & & -23.823 & 2.3712 & -26.092 & 2.3146 \\
\hline 3 & 0.1 & 5 & -12.224 & 2.5265 & -14.15 & 2.4049 \\
\hline & 0.15 & & -14.708 & 3.2264 & -18.384 & 2.9946 \\
\hline & 0.2 & & -17.755 & 4.1124 & -24.026 & 3.7209 \\
\hline 3 & 0.15 & 1 & -8.4326 & 3.5758 & -9.8259 & 3.4665 \\
\hline & & 2 & -10.364 & 3.4680 & -12.526 & 3.3159 \\
\hline & & 3 & -11.988 & 3.3774 & -14.740 & 3.1931 \\
\hline & & 7 & -16.998 & 3.1008 & -21.418 & 2.8347 \\
\hline & & & & & & \\
\hline
\end{tabular}

The impact of $\beta, \chi$, and $M$ on physical quantities, such as 
$\operatorname{Re}^{-1 / 2} \mathrm{Nu}$ and $\operatorname{Re}^{1 / 2} C_{f}$ have been summarized in table 3. In this table, we perceived that an increase in the three parameters, $\beta, \chi$, and $M$, leads to minimizing the local skin friction coefficient in the existence of CNTs nanoparticles suspended in water and human blood based fluids. Besides, as an increase in the $\beta$ and $M$, it leads to a decrease in

$\operatorname{Re}^{-1 / 2} \mathrm{Nu}$ of the CNTs nanoparticles based water and human blood. But the opposite case happens, when the $\chi$ is increased, then leads to an increment in $\operatorname{Re}^{-1 / 2} N u$ and $\operatorname{Re}^{1 / 2} C_{f}$. Besides, it has been observed from the table that CNTs/Human blood has a high $\operatorname{Re}^{-1 / 2} N u$ and $\operatorname{Re}^{1 / 2} C_{f}$ compared with CNTs/water Casson nanofluid. In addition, SWCENT has lower $\operatorname{Re}^{1 / 2} C_{f}$ than MWCENT based Human blood Casson nanofluid, but the opposite case happens in water-based Casson nanofluid. Also, SWCENT human blood/water has high $\operatorname{Re}^{-1 / 2} N u$ compared with MWCENT human blood/water.

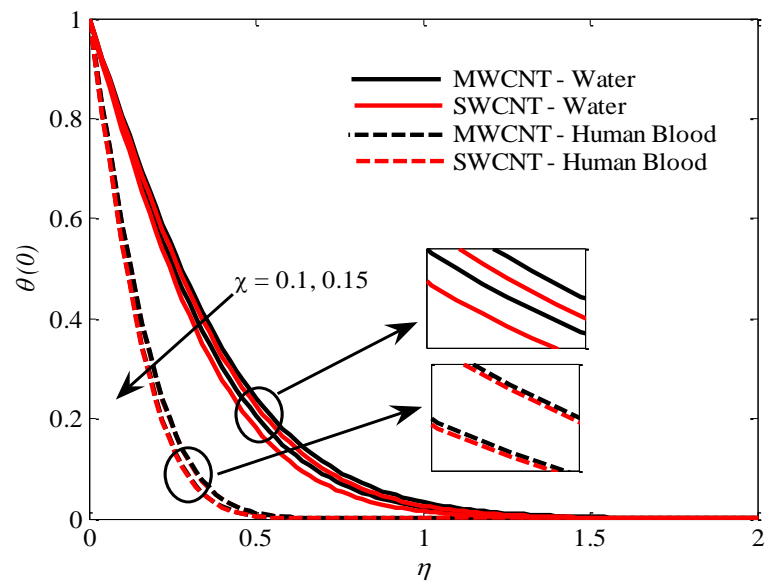

Fig. 2 Temperature profile vs $\chi$.

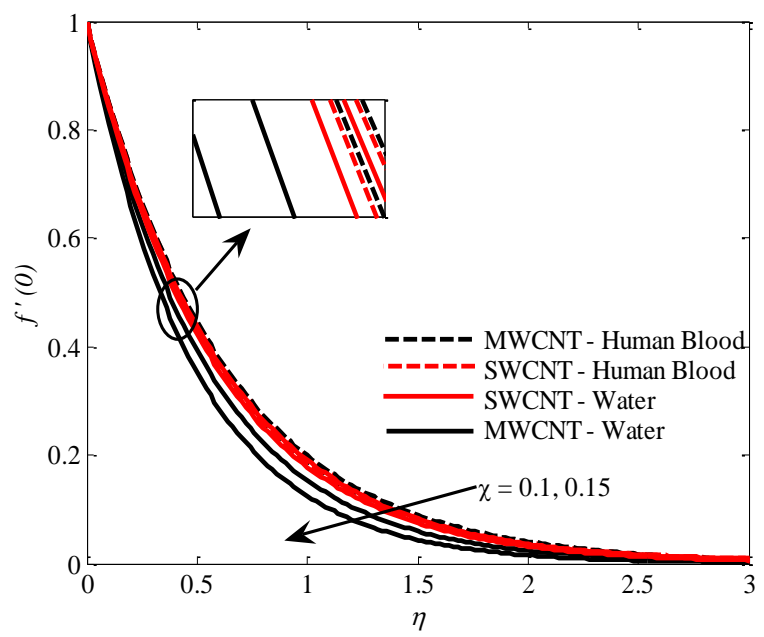

Fig. 3 Velocity profile vs $\chi$.

Figures 2 and 3 show that both results of the quantities of the temperature and velocity are reduced with an increment of values of the $\chi$. Moreover, it is noted that CNTs/human blood has a lower temperature than CNTs/water, but the reverse case happens in the velocity profile with an increase in the value of thermal boundary layer thickness. Also, the MWCENT nanoparticle has a higher temperature opposite to SWCNT nanoparticles, and the reason is that the density of SWCNT is greater than it of MWCNT. Moreover, it can be seen that SWCNT/human blood has a lower velocity than MWCENT/human blood with an increment of the value of thermal boundary layer thickness, but the opposite case happens in MWCENT and SWCNT that are suspending in water.

Figures 4 and 5 exhibit that when the $M$ increase, then the temperature increase, while the velocity profiles are decrement. Practically it has been noticed that the presence of a magnetic field in the flow field region decelerates the fluid motion. The resistive force created is known as the Lorentz force. So, the Lorentz force slows down the velocity of the fluid. In addition, human blood has high temperatures and low velocity compared to water with an increase in the values of the $M$. Moreover, MWCENT has a high velocity and temperature than SWCENT suspended in human blood Casson nanofluid, meanwhile, when the CNTs in water, we noticed that the SWCENT has a lower temperature than MWCENT nanoparticles, but the reverse case happens in the velocity profile.

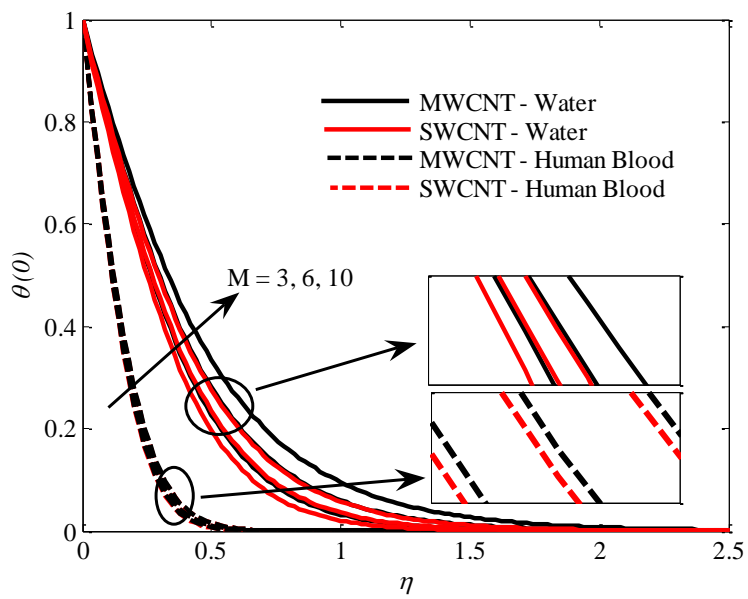

Fig. 4 Temperature profile vs $M$.

Figures 6 and 7 display the effects of the $\beta$ on the temperature and velocity profiles of Casson nanofluid. It is observed that the increasing in $\beta$ values, leads to increasing in the temperature and decreasing in the velocity profiles, in the presence of CNTs suspended in human blood/water Casson nanofluid. Furthermore, it can be clearly seen that the MWCENT has high temperature than SWCENT nanoparticles. Besides, the MWCENT has less velocity than SWCENT in water, but the opposite case happens when the CENTs nanoparticles suspended in human blood based Casson nanofluid, the MWCENT has high velocity than SWCENT in human blood. On the other hand, human blood has high velocity and less temperature compared with water.

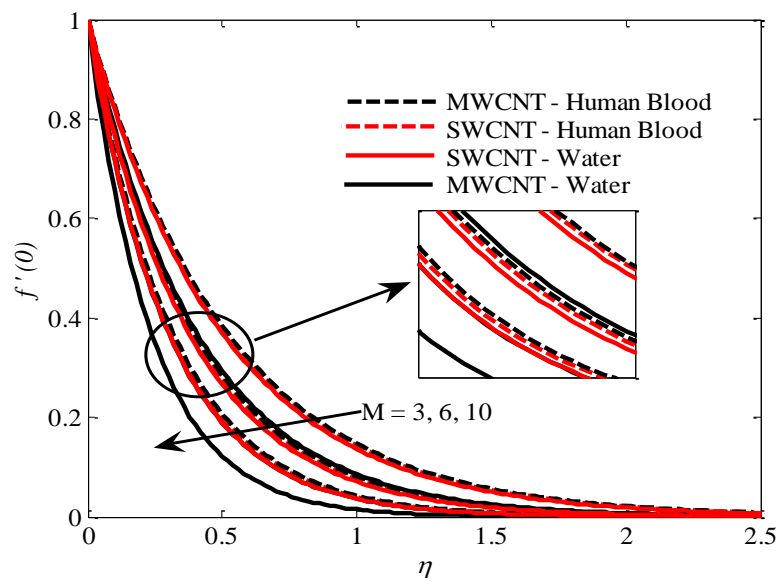

Fig. 5 Velocity profile vs $M$. 


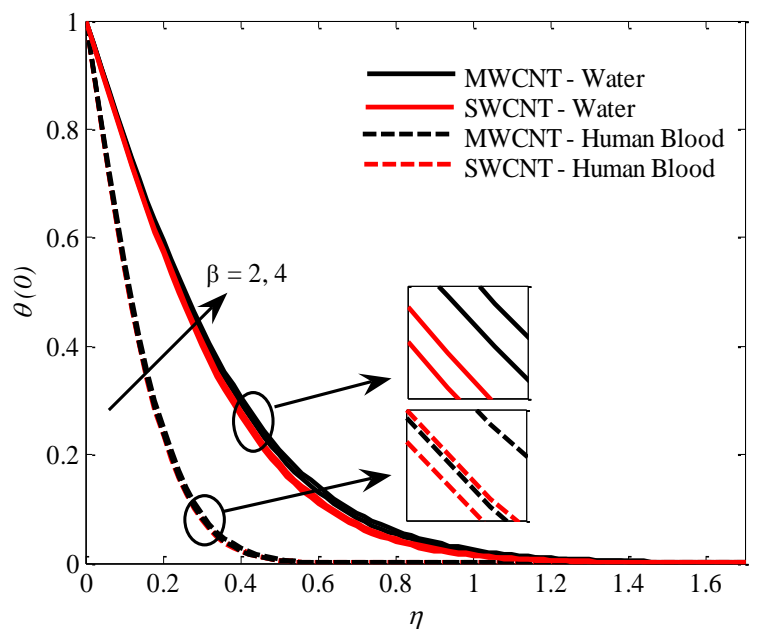

Fig. 6 Temperature profile vs $\beta$.

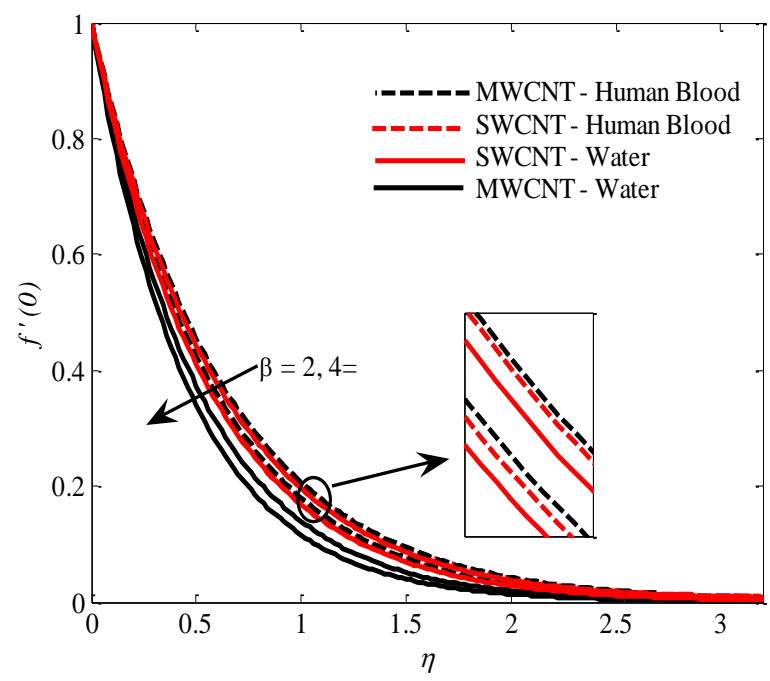

Fig. 7 Velocity profile vs $\beta$.

\section{CONCLUSIONS}

This paper offers a facilitated mathematical model and numerically studies the heat transfer effects for free convection in MHD Casson nanofluid flow over a stretching sheet. Several properties of the MHD Casson nanofluid parameters on the physical quantities, such as the local skin friction coefficient and the local Nusselt number, as well as temperature and velocity are considered in an attempt to analyse them through various plots and tables. In addition the constant wall temperature boundary condition has been considered in this study. The following results are the findings of the study:

1. The temperature profile of water is more than it for human blood based Casson nanofluid when the values of Casson, magnetic, and nanoparticle volume fraction parameters are increased.

2. When MHD Casson nanofluid parameters increase, local skin friction coefficient in SWCENT/human blood is less than it in MWCENT/human blood based Casson nanofluid .

3. MWCENT human blood/water has a higher temperature profile compared with SWCENT human blood /water with an increase in the MHD Casson nanofluid parameters.

4. SWCENT has lower local skin friction than MWCENT based Human blood Casson nanofluid, but the opposite case happens in water-based Casson nanofluid. Also, SWCENT human blood/water has a high local Nusselt number compared with MWCENT human blood/water.

\section{ACKNOWLEDGEMENTS}

You may acknowledge the funding source of your work as well as suggestions and comments from, and discussions with colleagues in this section.

\section{NOMENCLATURE}

$B \quad$ Thermal expansion coefficient

$C_{f} \quad$ Local skin friction coefficient

$c_{\rho} \quad$ Specific heat

$g \quad$ Acceleration due to gravity

Gr Grashof number

$K \quad$ Material or micropolar parameter

$k \quad$ Thermal conductivity

$M \quad$ Magnetic parameter

$\mathrm{Nu} \quad$ Local Nusselt number

Pr Prandtl number

$p_{y} \quad$ Yield stress of the fluid

$q_{w} \quad$ Constant wall heat flux

$T \quad$ Fluid temperature

$T_{\infty} \quad$ Temperature of the ambient fluids

$T_{w} \quad$ Wall temperature

$\tilde{u}, \tilde{v} \quad$ Non-dimensional velocity components

along $\tilde{x}$ and $\tilde{y}$ directions

$\tilde{x}, \tilde{y} \quad$ Coordinates measured from the lower stagnation point along the surface of cylinder and Normal to it, respectively

\section{GREEK SYMBOLS}

$\alpha \quad$ Thermal diffusivity

$\beta \quad$ Parameter of the Casson fluid

$\theta \quad$ Non-dimensional temperature

$\mu \quad$ Dynamic viscosity

$\mu_{B} \quad$ Plastic dynamic viscosity of the non-Newtonian fluid

$\pi_{c} \quad$ Critical value of this product based on the

non-Newtonian model

$\kappa \quad$ Vortex viscosity

$\sigma_{f} \quad$ Electric conductivity

$\alpha \quad$ Thermal diffusivity

$\rho \quad$ Fluid density

$\chi \quad$ Volume fraction

$\psi \quad$ stream function

\section{REFERENCES}

Ahmed, T. N., and Khan, I., 2018, "Mixed convection flow of sodium alginate (SA-NaAlg) based molybdenum disulphide (MoS2) nanofluids: Maxwell Garnetts and Brinkman models", Results in Physics, 8,752-757. https://doi.org/10.1016/j.rinp.2018.01.004

Alkasasbeh, H. T., 2018., "Numerical solution of micropolar Casson fluid behaviour on steady MHD natural convective flow about a solid sphere", Journal of Advanced Research in Fluid Mechanics and Thermal Sciences, 50, 55-66. 
Alkasasbeh, H. T., Salleh, M. Z., Nazar, R., and Pop, I., 2014, "Numerical solutions of radiation effect on magnetohydrodynamic free convection boundary layer flow about a solid sphere with Newtonian heating", Applied Mathematical Sciences, 8, 6989-7000.

Alsagri, A. S., Nasir, S., Gul, T., Islam, S., Nisar, K., Shah, Z., and Khan, I., 2019, "MHD thin film flow and thermal analysis of blood with CNTs nanofluid", Coatings, 9, 175-190.

https://doi.org/10.3390/coatings9030175

Alwawi, F. A., Alkasasbeh, H. T., Rashad, A., and Idris, R., 2020, "MHD natural convection of Sodium Alginate Casson nanofluid over a solid sphere", Results in physics, 16, 102818.

https://doi.org/10.1016/j.rinp.2019.102818

Alwawi, F. A., Alkasasbeh, H. T., Rashad, A. M., and Idris, R., 2019, "Natural convection flow of Sodium Alginate based Casson nanofluid about a solid sphere in the presence of a magnetic field with constant surface heat flux", Journal of Physics: Conference Series, 1366012005 https://doi:10.1088/1742-6596/1366/1/012005

Aman, S., Khan, I., Ismail, Z., Salleh, M. Z., Alshomrani, A. S., and Alghamdi, M. S., 2017, "Magnetic field effect on Poiseuille flow and heat transfer of carbon nanotubes along a vertical channel filled with Casson fluid", AIP Advances, 7, 015036.

https://doi.org/10.1063/1.4975219

Bhattacharyya, K., 2013, "Boundary layer stagnation-point flow of casson fluid and heat transfer towards a shrinking/stretching sheet", Frontiers in Heat and Mass Transfer (FHMT), 4, 023003 https://dx.doi.org/10.5098/hmt.v4.2.3003

Casson, N., 1959, "A flow equation for pigment-oil suspensions of the printing ink type", Rheology of disperse systems.

Cebeci, and Bradshaw, P., 2012, "Physical and computational aspects of convective heat transfer", Springer Science \& Business Media.

Chamkha, and Aly, A., 2010, "MHD free convection flow of a nanofluid past a vertical plate in the presence of heat generation or absorption effects", Chemical Engineering Communications, 198,425441. https://doi.org/10.1080/00986445.2010.520232

Chamkha, Gorla, R. S. R., and Ghodeswar, K., 2011, "Non-similar solution for natural convective boundary layer flow over a sphere embedded in a porous medium saturated with a nanofluid", Transport in porous media, 86, 13-22. https://doi.org/10.1007/s11242-010-9601-0

Chamkha, A., 2011, "Natural convective boundary layer flow over a horizontal plate embedded in a porous medium saturated with a nanofluid", Journal of Modern Physics, 2011.

https://doi:10.4236/jmp.2011.22011

Choi, and Eastman, J. A., 1995, "Enhancing thermal conductivity of fluids with nanoparticles", Argonne National Lab., IL (United States).

Ding, Y., Alias, H., Wen, D., and Williams, R. A., 2006, "Heat transfer of aqueous suspensions of carbon nanotubes (CNT nanofluids)", International Journal of Heat and Mass Transfer, 49, 240-250 https://doi.org/10.1016/j.ijheatmasstransfer.2005.07.009

Ellahi, R., Alamri, S. Z., Basit, A., and Majeed, A., 2018, Effects of "MHD and slip on heat transfer boundary layer flow over a moving plate based on specific entropy generation", Journal of Taibah University for Science, 12, 476-482.

https://doi/full/10.1080/16583655.2018.1483795

Haq, R. U., Nadeem, S., Khan, Z. H., and Noor, N. F. M., 2015, "Convective heat transfer in MHD slip flow over a stretching surface in the presence of carbon nanotubes", Physica B: condensed matter, 457, 40-47 https://doi.org/10.1016/j.physb.2014.09.031
Hassanien, I., Abdullah, A., and Gorla, R., 1998, "Flow and heat transfer in a power-law fluid over a nonisothermal stretching sheet", Mathematical and computer modelling, 28, 105-116. https://doi.org/10.1016/S0895-7177(98)00148-4

Hayat, T., Shehzad, S., and Alsaedi, A., 2012, "Soret and Dufour effects on magnetohydrodynamic (MHD) flow of Casson fluid", Applied Mathematics and Mechanics, 33, 1301-1312. https://doi.org/10.1007/s10483-012-1623-6

Hussanan, A., Salleh, M. Z., Alkasasbeh, H. T., and Khan, I., 2018, "MHD flow and heat transfer in a Casson fluid over a nonlinearly stretching sheet with Newtonian heating", Heat Transfer Research, 49 , 1185-1198. https://doi.org/10.1615/HeatTransRes.2018014771

Iijima, S., 1991, "Helical microtubules of graphitic carbon", Nature, 354, 56-58. https://doi.org/10.1038/354056a0

Keller, and Bramble, J., 1970, "A new difference method for parabolic problems. Numerical Methods for Partial Differential Equations", Academic Press. New York. USA.

Khalid, A., Khan, I., Khan, A., Shafie, S., and Tlili, I., 2018, "Case study of MHD blood flow in a porous medium with CNTS and thermal analysis", Case studies in thermal engineering, 12, 374-380. https://doi.org/10.1016/j.csite.2018.04.004

Khan, W., Khan, Z., and Rahi, M., 2014, "Fluid flow and heat transfer of carbon nanotubes along a flat plate with Navier slip boundary", Applied Nanoscience, 4, 633-641.https://doi.org/10.1007/s13204-013$\underline{0242-9}$

Makinde, O., 2012, "Computational modelling of MHD unsteady flow and heat transfer toward a flat plate with Navier slip and Newtonian heating", Brazilian Journal of Chemical Engineering, 29, 159-166. https://doi.org/10.1590/S0104-66322012000100017

Mukhopadhyay, S., Bhattacharyya, K., and Hayat, T., 2013, "Exact solutions for the flow of Casson fluid over a stretching surface with transpiration and heat transfer effects", Chinese Physics B, 22, 114701. https://doi.org/10.1088/1674-1056/22/11/114701

Mustafa, M., Hayat, T., Ioan, P., \& Hendi, A., 2012, "Stagnation-point flow and heat transfer of a Casson fluid towards a stretching sheet", Zeitschrift für Naturforschung A, 67, 70-76.

https://doi.org/10.5560/zna.2011-0057

Mustafa, M., Hayat, T., Pop, I., and Aziz, A., 2011, "Unsteady boundary layer flow of a Casson fluid due to an impulsively started moving flat plate", Heat Transfer-Asian Research, 40, 563-576. https://doi.org/10.1002/htj.20358

Nadeem, S., Haq, R. U., and Akbar, N. S., 2013, "MHD threedimensional boundary layer flow of Casson nanofluid past a linearly stretching sheet with convective boundary condition", IEEE Transactions on Nanotechnology, 13, 109-115. https://doi.org/10.1109/TNANO.2013.2293735

Nadeem, S., Haq, R.U., Akbar, N.S., and Khan, Z. H., 2013, "MHD threedimensional Casson fluid flow past a porous linearly stretching sheet”, Alexandria Engineering Journal, 52, 577-582.

https://doi.org/10.1016/j.aej.2013.08.005

Nasir, S., Islam, S., Gul, T., Shah, Z., Khan, M. A., Khan, W., Khan, S., 2018, "Three-dimensional rotating flow of MHD single wall carbon nanotubes over a stretching sheet in presence of thermal radiation", Applied Nanoscience, 8, 1361-1378. https://doi.org/10.1007/s13204018-0766-0

Palani, G., and Srikanth, U., 2009, "MHD flow past a semi-infinite vertical plate with mass transfer", Nonlinear Anal.: Modell. Control, 14, 345-356. 
Prasad, V. R., Vasu, B., Bég, O. A., and Parshad, R. D., 2012, “Thermal radiation effects on magnetohydrodynamic free convection heat and mass transfer from a sphere in a variable porosity regime", Communications in Nonlinear Science and Numerical Simulation, 17, 654-671. https://doi.org/10.1016/j.cnsns.2011.04.033

Qadan, H., Alkasasbeh, H., Yaseen, N., Sawalmeh, M. Z., and Alkhalafat, S., 2019, "A Theoretical study of steady MHD mixed convection heat transfer flow for a horizontal circular cylinder embedded in a micropolar casson fluid with thermal radiation", Journal of Computational Applied Mechanics, 50, 165-173. https://doi.org/10.22059/JCAMECH.2019.278376.372

Qing, J., Bhatti, M., Abbas, M., Rashidi, M., and Ali, M., 2016, "Entropy generation on MHD Casson nanofluid flow over a porous stretching/shrinking surface", Entropy, 18, 123.

https://doi.org/10.3390/e18040123

Rashidi, M. M., Bhatti, M. M., Abbas, M. A., and Ali, M. E. S., 2016, "Entropy generation on MHD blood flow of nanofluid due to peristaltic waves", Entropy, 18, 117-132. https://doi.org/10.3390/e18040117

Sallam, A. M., and Hwang, N. H., 1984, "Human red blood cell hemolysis in a turbulent shear flow: contribution of Reynolds shear stresses", Biorheology, 21, 783-797. https://doi.org/10.3233/BIR-1984$\underline{21605}$

Salleh, M. Z., Nazar, R., and Pop, I., 2010, "Boundary layer flow and heat transfer over a stretching sheet with Newtonian heating", Journal of the Taiwan Institute of Chemical Engineers, 41, 651-655. https://doi.org/10.1016/j.jtice.2010.01.013

Seddeek, M., 2002, "Effects of radiation and variable viscosity on a MHD free convection flow past a semi-infinite flat plate with an aligned magnetic field in the case of unsteady flow", International journal of heat and mass transfer, 45, 931-935. https://doi.org/10.1016/S0017-9310(01)00189-2

Sheikholeslami, M., Gorji-Bandpy, M., and Vajravelu, K., 2015, "Lattice Boltzmann simulation of magnetohydrodynamic natural convection heat transfer of $\mathrm{Al} 2 \mathrm{O} 3$-water nanofluid in a horizontal cylindrical enclosure with an inner triangular cylinder", International journal of heat and mass transfer, 80, 16-25

https://doi.org/10.1016/j.ijheatmasstransfer.2014.08.090

Subba Rao, A., Nagaraja, L., Sudhakar Reddy, M., and Surya Narayana Reddy, M., 2017, "Steady-state transport phenomena on induced magnetic field modellinf for non-newtonian tangent hyperbolic fluid from an isothermal sphere with soret and dufour effects", Frontiers in Heat and Mass Transfer (FHMT), 9. 1-11

http://dx.doi.org/10.5098/hmt.9.17

Swalmeh M. Z, Alkasasbeh, H. T., Hussanan, A., Nguyen Thoi, T., and Mamat, M., 2019a, "Microstructure and inertial effects on natural convection micropolar nanofluid flow about a solid sphere", International Journal of Ambient Energy, 40, 1-31. https://doi.org/10.1080/01430750.2019.1665582

Swalmeh, M. Z., Alkasasbeh, H. T., Hussanan, A., and Mamat, M., 2019b, "Numerical Investigation of Heat Transfer Enhancement with Ag-GO Water and Kerosene Oil Based Micropolar Nanofluid over a Solid Sphere", Journal of Advanced Research in Fluid Mechanics and Thermal Sciences, 59, 269-282.

Tahir, F., Gul, T., Islam, S., Shah, Z., Khan, A., Khan, W., and Ali, L., 2017, "Flow of a nano-liquid film of Maxwell fluid with thermal radiation and magneto hydrodynamic properties on an unstable stretching sheet”, Journal of Nanofluids, 6, 1021-1030. https://doi.org/10.1166/jon.2017.1400

Tiwari, and Das, M. K., 2007, "Heat transfer augmentation in a twosided lid-driven differentially heated square cavity utilizing nanofluids", International journal of heat and mass transfer, 50, 20022018. https://doi.org/10.1016/j.ijheatmasstransfer.2006.09.034

Upreti, H., Pandey, A. K., and Kumar, M., 2018, "MHD flow of Agwater nanofluid over a flat porous plate with viscous-Ohmic dissipation, suction/injection and heat generation/absorption", Alexandria engineering journal, 57, 1839-1847.

https://doi.org/10.1016/j.aej.2017.03.018 